\title{
Plano Diretor de Tecnologia da Informação e Comunicação: objetivos estratégicos orientados pela e-governança
}

\author{
Ana M. M. de Paiva' ${ }^{1}$, Eunice P. S. Nunes ${ }^{2}$, Cristiano Maciel $^{2}$ \\ 1Secretaria de Tecnologia da Informação \\ 2Instituto de Computação \\ Universidade Federal de Mato Grosso (UFMT) \\ 78060-900 - Cuiabá-MT - Brasil \\ \{anamarquespaiva, eunice\}@ufmt.br,crismac@gmail.com
}

\begin{abstract}
Resumo. As Tecnologias da Informação e Comunicação são fundamentais para o aumento do desempenho da administração pública. Logo, estratégias de governança eletrônica, que buscam direcionar, monitorar e avaliar as instituições, são essenciais para modernizar os processos internos e melhorar os serviços públicos entregues ao cidadão. Assim, este trabalho apresenta a metodologia de construção do Plano Diretor de Tecnologia da Informação e Comunicação (PDTIC) na Universidade Federal de Mato Grosso (UFMT). Há indícios que um PDTIC construído com engajamento da alta administração do órgão, colaboração da comunidade interna e aplicação de métodos de priorização, pode contribuir para o alcance dos objetivos organizacionais.
\end{abstract}

Abstract. Technologies of Information and Communication are fundamental for increasing the performance of public administration. Therefore, e-governance strategies, which seek to direct, monitor and evaluate institutions, are essential to modernize internal processes and improve public services delivered to citizens. Thus, this work presents the construction methodology of the Master Plan for Information and Communication Technology (PDTIC) at the Federal University of Mato Grosso (UFMT). There are indications that a PDTIC built with the engagement of the organ's top management, collaboration of the internal community and application of prioritization methods, can contribute to the achievement of organizational objectives.

\section{Introdução}

O constante avanço tecnológico e o bom uso dos recursos públicos, em consonância com a Governança Digital de um órgão, demandam maior planejamento e gestão dos recursos de Tecnologia da Informação e Comunicação (TIC). Neste âmbito, a TIC tem por foco a efetiva utilização da informação como suporte às diretrizes e objetivos organizacionais.

No Brasil, práticas de governo eletrônico (e-gov) vêm sendo introduzidas desde os anos 2000 [Przeybilovicz et al. 2018]. As ações de e-gov (do inglês eletronic government) tem a finalidade de priorizar e democratizar o acesso à informação com o uso das TICs, "visando ampliar o debate e a participação popular na construção das políticas públicas, e, também, aprimorar a qualidade e a efetividade dos serviços e informações." [BRASIL 2018, p.13]. Desde então, diversas ações da Administração 
Pública Federal brasileira foram sendo desenvolvidas e culminaram na evolução do paradigma de "governo eletrônico" para "governo digital” [BRASIL 2018].

Nesse cenário, o planejamento de TIC é uma das ações de governança digital, que visa direcionar os recursos de TIC, a fim de alcançar as metas e objetivos organizacionais [UFMT 2019a]. Deste modo, no planejamento identificam-se soluções de TIC que permitem o aprimoramento do negócio, definindo iniciativas de curto, médio e longo prazo. Logo, o Plano Diretor de Tecnologia da Informação e Comunicação (PDTIC) apresenta-se como uma das estratégias de governança digital, a fim de orientar a execução dos projetos de TIC, justificar o investimento e oferecer indicadores que visem melhorar as práticas de controle, gerando valor para a organização e a sociedade, por meio da entrega de serviços digitais ao cidadão.

Nesta perspectiva, o planejamento de TIC deve ser elaborado de maneira participativa, incluindo as unidades finalísticas e a comunidade, neste caso a acadêmica, consolidando-se no PDTIC que, de acordo com a Instrução Normativa SGD/ME N ${ }^{o} 1$, de 4 de abril de $2019^{1}$, artigo $2^{\circ}$, inciso XXV, trata-se de um "instrumento de diagnóstico, planejamento e gestão dos recursos e processos de TIC, com o objetivo de atender às necessidades finalísticas e de informação de um órgão ou entidade para um determinado período" [BRASIL 2019a].

Ressalta-se que o PDTIC deve estar alinhado à Estratégia de Governança Digital (EGD) do Governo Federal, instituída pelo Decreto $\mathrm{n}^{\circ} 10.332$ de abril de 2020 [BRASIL 2020], e ao Plano de Desenvolvimento Institucional (PDI) [UFMT 2019b]. Portanto, deve contemplar indicadores e metas alinhados às necessidades governamentais e, estrategicamente, orientar as metas e iniciativas de TIC. Destaca-se ainda que o PDTIC atua em nome da comunidade universitária, moral e democraticamente [Gomes et al. 2018], atendendo aos princípios da transparência, deve ser público e passar por avaliações periódicas. Todavia, como se dá, na prática, a construção de um PDTIC?

Com foco neste questionamento, este trabalho objetiva gerar conhecimentos para a aplicação prática Gil [2019], dirigida à apresentar a metodologia de construção do PDTIC da Universidade Federal de Mato Grosso, para o período de 2020 a 2022, alinhado aos anseios da comunidade acadêmica, ao PDI 2019-2023 e à EGD 2020 a 2022. Para consecução do objetivo deste estudo, foram realizadas pesquisa documental, bibliográfica e estudo de caso único [Yin 2015], com abordagem qualitativa [Marconi \& Lakatos 2017]. As etapas e ferramentas usadas na elaboração do PDTIC são apresentadas na seção 3, a construção do PDTIC, após exploração da fundamentação teórico deste estudo (seção 2). O PDTIC 2020-2022 é, então, apresentado na seção 4, seguido das considerações finais e referências utilizadas nesta pesquisa.

\section{Fundamentação Teórica}

As possibilidades de discussão desta temática face a literatura são variadas, todavia, dado os limites de exposição do conteúdo, foca-se em abordar aspectos relacionados às TIC e a governança, e ao planejamento destas.

\footnotetext{
${ }^{1}$ A Instrução Normativa SGD/ME N ${ }^{\circ}$, de 4 de abril de 2019, tem parte da redação alterada pela Instrução Normativa SGD/ME n ${ }^{\circ} 202$, de 18 de setembro de 2019 [BRASIL 2019b] e Instrução Normativa SGD/ME N ${ }^{\circ}$ 31, de 23 de março de 2021 [BRASIL 2021]. Salienta-se que as alterações citadas não prejudicam o inciso citado.
} 
A governança pública pode ser definida como um conjunto de mecanismos de liderança, estratégia e controle, postos em prática para avaliar, direcionar e monitorar a gestão de modo a conduzir as políticas públicas e a prestação dos serviços à sociedade (Decreto $\mathrm{n}^{\circ} 9.203$ de 22 de novembro de 2017). Os mecanismos de governança de TIC devem ser aprovados e apoiados pela alta administração e cobrir toda a organização, de forma a sustentar suas estratégias e objetivos [Bianchi et al. 2017] [ISACA 2018].

Nesse contexto, as tecnologias contribuem com a eficiência e eficácia das operações e serviços públicos, internamente na adaptação às mudanças políticas e processos de governança, e, externamente, na convergência de serviços e na participação social. Assim, alcançar eficácia e eficiência não é apenas uma questão de capitalizar as capacidades de TIC [Gottschalk 2009], mas atingir níveis mais elevados de maturidade na gestão de tecnologia, informação e processos organizacionais.

Conforme preconizado pelo Sistema de Administração dos Recursos de Tecnologia da Informação [SISP 2017], a governança de TIC é um sistema em que o uso atual e futuro das TICs é dirigido e controlado, mediante avaliação e direcionamento de sua aplicação para dar suporte e monitorar a execução dos planos estratégicos e operacionais do órgão. A governança corporativa de TIC é exercida pelo conselho que supervisiona a implementação de processos, estruturas e mecanismos relacionais, baseados em responsabilidades definidas, alinhamento estratégico e geração de valor [ISACA 2018].

O esforço conjunto dos diversos atores - governo, universidade, sociedade - explica a necessidade de coordenação das ações utilizando-se de mecanismos de governança, como pode ser observado no PDTIC. Portanto, mecanismos de governança também incluem atores não governamentais para melhorar a prestação de serviços públicos, a governança democrática ou a tomada de decisões, o que contribui com a maturidade e a incorporação de tecnologias [Renteria et al. 2019].

A implantação do governo digital exige o uso estratégico da TIC apoiada pela egovernança, com os objetivos de modernizar e simplificar operações de governo, melhorar a eficiência administrativa, disponibilizar de serviços apropriados e aprimorar $\mathrm{o}$ relacionamento das diversas partes interessadas para aumentar a transparência e responsabilização sobre os processos e serviços de tecnologia das instituições [Alarabiat et al. 2018].

Neste sentido, se espera que a adoção de tecnologias permita a transformação de diferentes estruturas e processos da organização e ajudar o cumprimento de seus objetivos gerais e estratégicos da área de TIC. A maturidade da e-governança contribui com as organizações, revelando sua posição em comparação com as capacidades e outros recursos necessários para atingir os objetivos da organização [Renteria et al. 2019]. Ela fornece um senso de foco e direção para as estratégias de melhoria da organização e ajudam como base para avaliar o posicionamento atual em relação aos estágios desejados de adoção e uso de tecnologia.

Para Gonçalves et al. [2020], aproximar os níveis estratégicos da instituição nas discussões acerca das tecnologias seria uma forma de facilitar e incluir a TIC nas tomadas de decisão e planos estratégicos para que sejam consideradas as capacidades e inovação que a TIC pode oportunizar às diversas áreas. Ademais, a constituição federal dispõe que aquele que não planeja incorre em inobservância jurídica do disposto no caput do art. 37 da CF/1988, pois age contra o princípio da eficiência [BRASIL 1993] 
Logo, ao se observar o status atual como ponto de partida, é possível estabelecer as trilhas pelas quais deverá se percorrer ao estágio desejado e, posteriormente, planejar os investimentos em tecnologias de informação e sua implementação. Nesta perspectiva, mecanismos de e-governança são importantes porque orientam o cumprimento de metas comuns, seja aquelas oriundas do Governo Federal, ou as específicas da instituição [Renteria et al. 2019].

\section{A Construção do PDTIC}

A elaboração e implantação do planejamento se apresentam como ações essenciais para geração de valor no negócio, e, consequentemente, à sociedade. Para isso, a comunicação dos gestores deve ser clara, assim como é necessário o engajamento e compromisso de todos os envolvidos no processo. Assim, no setor público, o planejamento estratégico assume papel imprescindível, para a consecução dos objetivos e estratégias locais e de Estado, haja vista a complexidade desses negócios.

\subsection{Alinhamento estratégico}

A agenda de pesquisa sobre TI está mais relacionada a desenvolver sistemas e entender as consequências da tecnologia da informação (modelos, técnicas ou dispositivos) a partir de certos objetivos específicos e condições operacionais. Porém, uma parcela considerável da pesquisa sobre TI está na concepção, implementação e uso de artefatos, como o PDTIC, que representam soluções concretas para os problemas do mundo real. Para melhor compreensão do alinhamento estratégico existente no objeto de estudo, antes, deve-se observar o arranjo das organizações vinculadas ao Sistema de Administração dos Recursos de Tecnologia da Informação (SISP).

Instituído pelo Decreto $\mathrm{n}^{\circ} 7.579$, de 11 de outubro de 2011[BRASIL 2011], com o objetivo de organizar a operação, controle, supervisão e coordenação dos recursos de tecnologia da informação da administração direta, autárquica e fundacional do Poder Executivo Federal. É vinculado ao Ministério da Economia, tendo como órgão central a Secretaria de Governo Digital (SGD) da Secretaria Especial de Desburocratização, Gestão e Governo Digital., sendo responsável por diversos órgãos ${ }^{2}$ divididos entre: i) órgãos setoriais; ii) órgãos seccionais, e; iii) órgão correlatos.

Dentre as unidades de administração dos recursos de tecnologia da informação das autarquias e fundações os órgãos setoriais está a UFMT, por meio da Secretaria de Tecnologia da Informação e Comunicação, tendo como as principais atribuições:

1. Planejar, implantar e acompanhar a Política Institucional de Tecnologia da Informação e Comunicação, em consonância às diretrizes governamentais nacionais e recomendações internacionais.

2. Propor o Plano Diretor de Tecnologia da Informação (PDTI), em consonância com o Plano de Desenvolvimento Institucional e as políticas de governo.

3. Propor, desenvolver, implantar e acompanhar a execução de projetos relacionados à TIC, alinhada às necessidades da Instituição.

4. Apoiar a alta administração no Comitê de Governança Digital [UFMT 2019a, p.13]

Por fim, em respeito às normatizações aplicáveis no contexto das Universidades, destacam-se os seguintes documentos estruturantes: i) Plano de Desenvolvimento

\footnotetext{
${ }^{2}$ https://www.gov.br/governodigital/pt-br/sisp/sobre-o-sisp/orgaos-do-sisp
} 
Institucional (PDI); ii) Plano de Logística Sustentável (PLS); iii) Plano de Transformação Digital (PTD); iv) Plano Diretor de Tecnologias da Informação e Comunicação (PDTIC), e; v) Plano de Dados Abertos (PDA).

Acrescenta-se que para desenvolvimento dos planos e metas de Governo deve-se haver a sinergia entre os objetivos das instituições públicas. No âmbito da TIC, o alinhamento pode ser ilustrado de maneira simplificada, conforme a Figura 1.

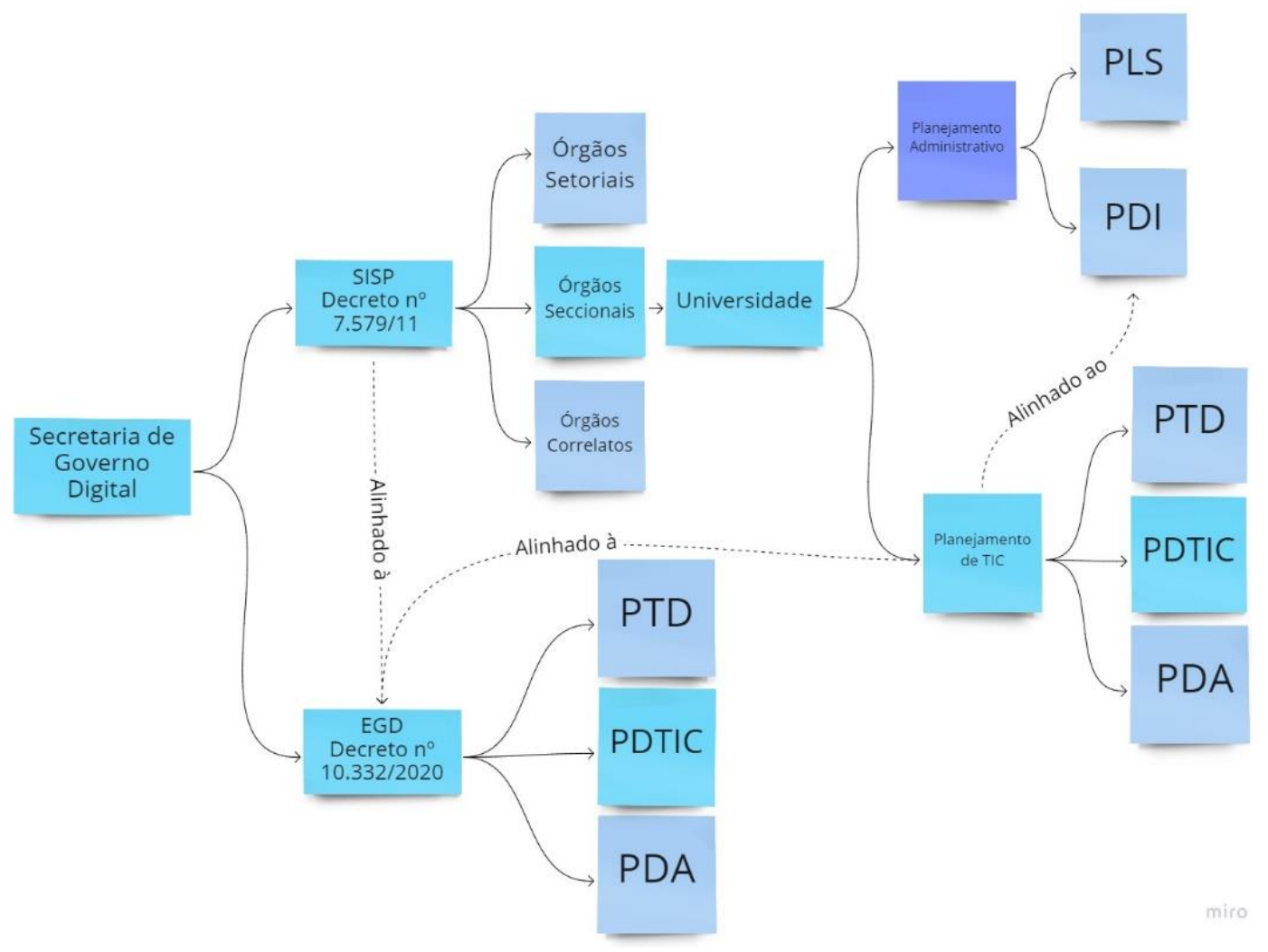

Figura 1 - Alinhamento estratégico do planejamento de TIC Fonte: dos autores [2021].

\subsection{O Processo de Construção do PDTIC}

Os macroprocessos relativos à elaboração do PDTIC 2020-2022 da UFMT são apresentados na Figura 2. Sua construção teve início com a criação de um Grupo de Trabalho (GT), a partir do Comitê de Governança Digital, com a participação dos gestores da Pró-Reitoria de Ensino de Graduação, da Pró-Reitoria de Planejamento e da Secretaria de Tecnologia da Informação, bem como das Pró-Reitorias dos campi de Sinop, Araguaia e Várzea Grande, além da Universidade Federal de Rondonópolis, tendo em vista a tutoria da UFMT sobre essa instituição. Cada gestor fez a indicação de representantes da área técnica para condução dos trabalhos. 


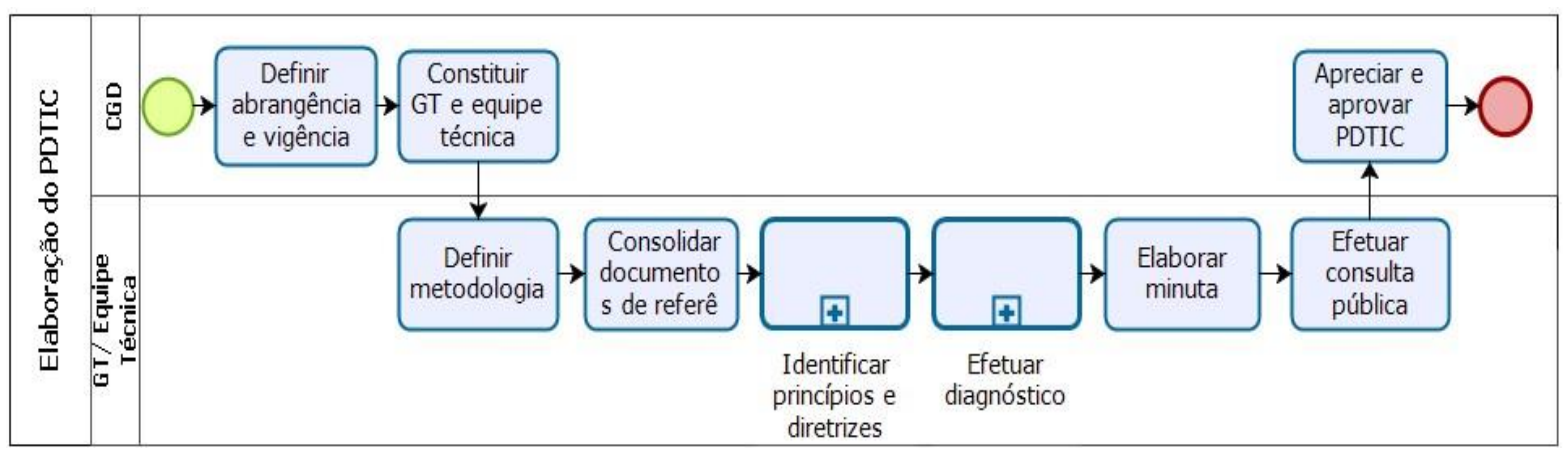

Figura 2 - Processo de elaboração do PDTIC Fonte: dos autores [2021].

Inicialmente, foi solicitada a participação às unidades acadêmicas e administrativas com contribuições das necessidades de TIC, por meio de Ofício Circular protocolado eletronicamente. Sua consolidação serviria de base à identificação dos princípios e diretrizes do PDTIC.

$\mathrm{Na}$ etapa seguinte, os trabalhos focaram na construção do diagnóstico, que envolveu três fases principais: i) diagnóstico do Planejamento Estratégico de Tecnologia da Informação (PETI) 2013-2019; ii) elaboração do inventário de necessidades institucionais para o próximo período; e iii) levantamento dos objetivos e metas a serem seguidos nos próximos três anos. Destaca-se que o período de três anos foi deliberado pelo Comitê de Governança Digital da UFMT, uma vez que a Portaria $n^{\circ} 778$ de 04 de abril de $2019^{3}$, publicada pela Secretaria de Governo Digital do Ministério da Economia, exige uma vigência mínima de dois anos com revisão anual.

Posteriormente, a partir do agrupamento em eixos do inventário de necessidades e da Matriz SWOT, uma ferramenta que permite que seja feita a inventariação das forças e fraquezas da TIC na universidade e as oportunidades e ameaças do ambiente com a finalidade em aperfeiçoar os recursos e competências de forma a transformar as aparentes ameaças em novas oportunidades [Flores 2021], as metas e os indicadores foram estruturados, refletindo: i) estratégias que demandam informações, atendidas por recursos de TIC, e; ii) recursos de TIC que atendem às necessidades de informação, alinhadas às estratégias (Figura 3).

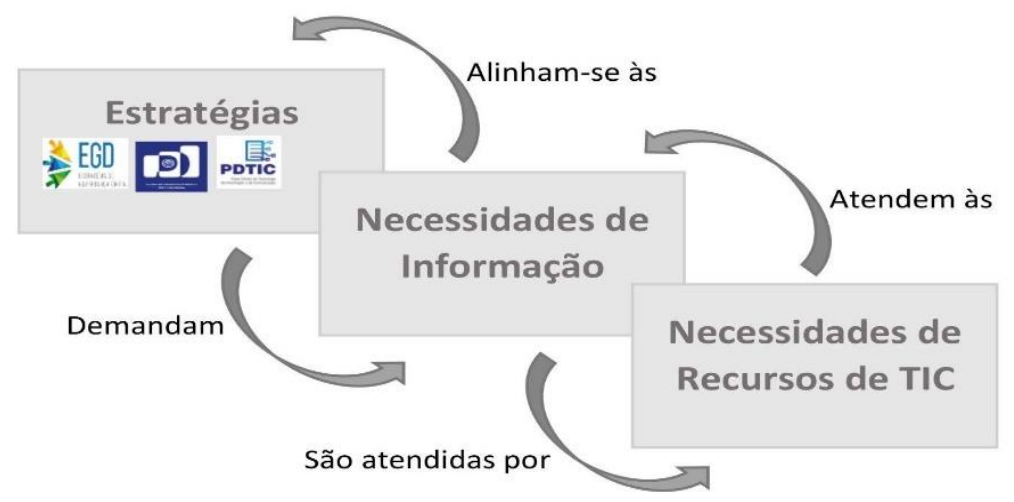

Figura 3 - Alinhamento estratégico Fonte: adaptada [BRASIL 2016, p.26]

3 https://www.in.gov.br/materia///asset publisher/Kujrw0TZC2Mb/content/id/70268218/do1-2019-04-05portaria-n-778-de-4-de-abril-de-2019-70268126 
Acrescenta-se que, neste momento foi realizado o alinhamento das metas e indicadores ao Plano de Desenvolvimento Institucional 2019-2023 da UFMT, à Estratégia de Governança Digital do Governo Federal e aos indicadores previstos no recredenciamento institucional, previsto para 2021, conforme ilustra a Figura 3. Destaca-se que, durante a elaboração do PDTIC da UFMT, o Governo Federal realizava consulta pública para o estabelecimento da EGD 2020-2021, a qual tem por propósito orientar e integrar as iniciativas de transformação digital no Poder Executivo Federal.

Por orientação do SISP, adotou-se a Matriz de Priorização de GUT (Gravidade x Urgência x Tendência) como ferramenta para solução de problemas, desenvolvida por Kepner e Tregoe. É um instrumento de qualidade usado para definir prioridades dadas as diversas alternativas de ação, uma vez que não é possível agir em diversas frentes simultaneamente [Kepner and Tregoe 1981]. A Matriz GUT com os parâmetros definidos pela equipe técnica de elaboração do PDTIC é apresentada na Tabela 1, em que cada um dos parâmetros é pontuado com peso de 1 a 5. A multiplicação dos parâmetros $(\mathrm{G}$ x U x T) indica o nível de prioridade das necessidades. Com a minuta do PDTIC estruturada, o CGD foi convocado para apreciação e aprovação do documento preliminar.

Tabela 1 - Matriz de Prioridade GUT

\begin{tabular}{|c|c|c|c|}
\hline Peso & Gravidade (G) & Urgência (U) & Tendência (T') \\
\hline 5 & Solução estratégica. & $\begin{array}{l}\text { Exigência legal com } \\
\text { prazo inferior a } 3 \text { meses } \\
\text { ou serviços contínuos. }\end{array}$ & $\begin{array}{l}\text { Interrompe a prestação } \\
\text { dos serviços. }\end{array}$ \\
\hline 4 & $\begin{array}{l}\text { Impacta os processos } \\
\text { rotinas administrativas ou } \\
\text { educacionais da UFMT. }\end{array}$ & $\begin{array}{l}\text { Exigência legal com } \\
\text { prazo de } 3 \text { a } 6 \text { meses. }\end{array}$ & $\begin{array}{l}\text { Impede a prestação dos } \\
\text { serviços. }\end{array}$ \\
\hline 3 & $\begin{array}{l}\text { Impacta os sistemas, } \\
\text { arquitetura de hardware, } \\
\text { dentre outros serviços de } \\
\text { TI. }\end{array}$ & $\begin{array}{l}\text { Tempo para } \\
\text { implementação entre } 6 \text { e } \\
12 \text { meses. }\end{array}$ & $\begin{array}{l}\text { Impede o cumprimento de } \\
\text { prazos para prestação dos } \\
\text { serviços. }\end{array}$ \\
\hline 2 & $\begin{array}{llr}\text { Impacta a gestão } & \text { e } \\
\text { desenvolvimento } & & \text { de } \\
\text { pessoas. } & & \\
\end{array}$ & $\begin{array}{lr}\text { Tempo } & \text { para } \\
\text { implementação entre } 12 \\
\text { e } 24 \text { meses. }\end{array}$ & $\begin{array}{l}\text { Dificulta a prestação dos } \\
\text { serviços. }\end{array}$ \\
\hline $\mathbf{1}$ & $\begin{array}{l}\text { Impacta melhorias } \\
\text { pontuais. }\end{array}$ & $\begin{array}{lr}\text { Tempo } & \text { para } \\
\text { implementação } & \text { superior } \\
\text { a } 24 \text { meses. } & \\
\end{array}$ & $\begin{array}{l}\text { Não interfere na prestação } \\
\text { dos serviços. }\end{array}$ \\
\hline
\end{tabular}

Fonte: PDTIC 2020-2022 [UFMT 2019a, p.75]

Em seguida, realizou-se a consulta pública para maior transparência e participação da comunidade, a qual ficou disponível em site próprio ${ }^{4}$ por 15 dias. Após consolidação das contribuições advindas da consulta pública, o documento final do PDTIC 2020-2022 foi submetido a Secretaria de Tecnologia da Informação que encaminhou ao CGD.

\section{Plano Diretor de Tecnologias da Informação e Comunicação}

O PDTIC 2020-2022, aprovado pelo CGD da UFMT, encontra-se público no Portal da UFMT $^{5}$ e, segue as orientações que constam na Portaria $n^{\circ} 778$ de 04 de abril de 2019 . O PDTIC é composto por: a) inventário de necessidades priorizado; b) plano de metas e ações; c) plano de gestão de pessoas; d) plano orçamentário; e) plano de gestão de riscos; f) monitoramento e revisão. O inventário de necessidades contribui, direta ou indiretamente, para alcançar os objetivos institucionais que se relacionam com a TIC. No documento do 
PDTIC 2020-2022, o inventário é estruturado na tabela a seguir, como ilustra a Figura 4 neste trabalho.

\begin{tabular}{|c|c|c|c|c|c|c|c|c|}
\hline \multirow{2}{*}{ ID } & \multicolumn{2}{|c|}{ Alinhamento Estratégico } & \multirow{2}{*}{ Eixo } & \multirow{2}{*}{ Necessidade } & \multicolumn{4}{|c|}{ Matriz de Prioridade } \\
\hline & PDI & EGD & & & G & $\mathrm{U}$ & $\mathrm{T}$ & GxUxT \\
\hline N01 & $\begin{array}{l}\text { Eixo: Gestão OBJ: } \\
02.01 ; 07.02 ; 09.03\end{array}$ & $\begin{array}{l}\text { Eixo: Ed. e Cap. Prof. } \\
\text { OBJ: } 10.35 ; 10.37\end{array}$ & $\begin{array}{|ll|}\text { Educação } & \text { e } \\
\text { Capacitação } & \\
\text { Profissional } & \\
\end{array}$ & $\begin{array}{l}\text { Dispor de pessoal qualificado } \\
\text { para atuação nas áreas } \\
\text { técnicas especificas }\end{array}$ & 4 & 3 & 4 & 48 \\
\hline
\end{tabular}

Figura 4 - Ilustração da Tabela 4 no PDTIC

Fonte: PDTIC 2020-2022 [UFMT 2019a, p.26]

No Plano de Metas e Ações estão descritas as metas para cada eixo, com seus respectivos objetivos estratégicos. Recomenda-se que as ações alinhadas aos eixos e objetivos, sejam consideradas no planejamento estratégico anual da área de TIC. Acerca do Plano Orçamentário, a Instrução Normativa Nº 1, de 4 de abril de 2019 [BRASIL 2019] dispõe que as contratações de TIC devem estar previstas no PDTIC e registradas no Plano Anual de Contratações (PAC). Quanto ao Plano de Gestão de Pessoas, o PDTIC apresenta o quadro de pessoal da STI, bem como o planejamento de incentivo à qualificação dos servidores lotados na área central de TIC. Quanto ao Plano de Riscos, a metodologia de gestão de riscos está relacionada aos objetivos estratégicos da UFMT definidos no PDI, mediante o tratamento adequado dos riscos pelos controles internos e o compartilhamento de informações essenciais para a tomada de decisões. Dessa forma, definiu-se que os riscos da TIC diretamente relacionados ao PDTIC serão priorizados, uma vez que já se encontra alinhado ao PDI 2019-2023 e ao Plano de Gestão de Riscos da unidade.

Por fim, o monitoramento e revisão do PDTIC, deve ser realizado anualmente, por meio de uma comissão composta por integrantes da STI lotados nos campi, que estabelecerá diálogo transversal com os diversos atores envolvidos na execução das metas propostas e de eventuais sugestões de alteração, mediante apresentação de justificativa e aprovação do CGD.

\section{Considerações finais}

Os resultados mostram que um PDTIC construído a partir de estratégias que incluem, principalmente, o engajamento da alta administração do órgão, a colaboração da comunidade interna (servidores e estudantes) e a aplicação de métodos de priorização, vislumbram maiores chances de alcançar os objetivos institucionais do órgão. Salienta-se que os apontamentos recebidos durante a consulta pública, foram contempladas no PDTIC em quatro eixos principais, cada qual com seus objetivos, indicadores e metas, que refletem as demandas da instituição para um período de três anos (2020-2022).

A governança e gestão de TIC na UFMT é coordenada pela STI. Entretanto, o processo de construção das políticas relativas à TIC é coletivo, apoiado pelo Comitê de Governança Digital e articula-se com a cooperação mútua e a racionalização de processos e de recursos, sempre com o foco em resultados e na geração de valor para a comunidade universitária.

O alinhamento estratégico entre os planos e metas de Governo e os documentos estruturantes da Universidade, orientados por mecanismos de e-governança, contribuem com o desenvolvimento regional e nacional. Além disso, permite a melhor aplicação dos recursos públicos, além do atendimento os princípios da eficiência, eficácia e economicidade, ao minimizar os riscos do negócio por meio do planejamento, 
monitoramento e eventuais correções no plano, por meio do diálogo transversal com os diversos atores envolvidos na execução das metas e ações.

Ademais, o PDTIC vigente na UFMT alavanca a Governança Digital no órgão e possibilita a alta administração direcionar, monitorar e avaliar os projetos e operações de TIC, de forma a identificar se caminham alinhados aos objetivos institucionais da UFMT e do Brasil.

A socialização de metodologias como essa pode apoiar outras instituições, em especial as de ensino superior, no planejamento de sua governança. Por fim, cabe salientar que é necessário o registro de tudo que tange à execução deste PDTIC, podendo redundar em trabalhos futuros que beneficiem a instituição e a sociedade.

\section{Referências}

Alarabiat, A., Soares, D., Ferreira, L. \& Sá-Soares, F. (2018). Analyzing E- Governance Assessment Initiatives: An Exploratory Study. In Proceedings of the 19th International Conference of the Digital Government Society Delft (Dg.o '18), 10 p.

Bianchi, I. S., Sousa, R. D., Pereira, R., Luciano, E. (2017). IT Governance Structures in Brazilian, Dutch and Portuguese Universities. Procedia Computer Science, 121, $927-$ 933.

BRASIL (1993). Lei $n^{\circ}$ 8.666, de 21 de junho de 1993. Lei de licitações e contratos administrativos. Regulamenta o artigo 37, inciso XXI, da Constituição Federal. Brasília: Câmara dos Deputados, 1993.

(2011). Decreto ${ }^{0}$ 7.579, de 11 de outubro de 2011. Dispõe sobre o Sistema de Administração dos Recursos de Tecnologia da Informação - SISP. Brasília - DF: Casa Civil. Presidência da República.

. (2016). Guia de PDTIC do SISP v2.0. Sistema de Administração dos Recursos de Tecnologia da Informação (SISP), Brasília - DF. Disponível em: http://www.sisp.gov.br/guiapdti/wiki/download/file/Guia de PDTI do SISP v2 Beta.pdf.

. (2018). Estratégia de Governança Digital: Transformação Digital - cidadania e governo. Ministério do Planejamento, Desenvolvimento e Gestão, Secretaria de Tecnologia da Informação e Comunicação, Brasil. Disponível em: https://www.gov.br/governodigital/pt-br/estrategia-de governancadigital/revisaodaestrategiadegovernancadigital20162019.pdf.

(2019a) Ministério da Economia. Secretaria de Governo Digital. Instrução Normativa SGD/ME No 1, de 4 de abril de 2019, Brasília-DF.

(2019b) Ministério da Economia. Secretaria de Governo Digital. Instrução Normativa SGD/ME n ${ }^{\circ}$, de 1 de abril de 2019, com redação alterada pela Instrução Normativa SGD/ME $\mathrm{n}^{\mathbf{0}}$ 202, de 18 de setembro de 2019. Disponível em: https://www.gov.br/governodigital/pt-br/contratacoes/in-01-de-2019compilada.pdf/view

. (2020). Decreto $n^{0} 10.332$, de abril de 2020. Estratégia de Governo Digital 20202022. Brasília - DF: Presidente da República, 2020. 
. (2021) Ministério da Economia. Secretaria de Governo Digital. Instrução Normativa $\mathrm{N}^{\mathrm{o}} 31$, de 23 de março de 2021, que altera a Instrução Normativa ${ }^{\mathrm{o}} 1$, de 4 de abril de 2019.

Flores, Felipe et al (2021). Aplicação das matrizes SWOT e GUT como planejamento estratégico na produção de carne bovina na fazenda Piquiá - Tomé-Açu, Amazônia, Pará, Brasil. Revista Gestão em Conhecimento, 5(5), fev. 2021.

Gil, A. C. (2019). Como Elaborar Projetos de Pesquisa. 6. ed. São Paulo: Atlas.

Gomes, W., Amorim, P. K. D. F., \& Almada, M. P. (2018). Novos desafios para a ideia de transparência pública. E-Compós, 21(2).

Gonçalves, D. M. S., Leal, A. L. C., Macedo, F. C., \& Castro, M. C. D. E. (2020). Modelagem orientada a objetivo como suporte à gestão estratégica de TI nos Institutos Federais de Educação. Revista de Gestão e Secretariado, 1(1), 176-200.

Gottschalk, P. (2009). Maturity levels for interoperability in digital government. Government Information Quarterly. 26, 1, 75-81.

ISACA. (2018). COBIT 2019 Framework: Introduction and Methodology. Rolling Meadows, IL: ISACA.

Kepner, C. H. and Tregoe, B. B. (1981). O administrador racional. São Paulo: Atlas.

Marconi, M. de A. \& Lakatos, E. M. (2017). Metodologia de Trabalho Científico, $8^{\text {a }}$ ed. Editora: Atlas.

Przeybilovicz, E., Cunha, M. A., and de Souza Meirelles, F. (2018). O uso da tecnologia da informação e comunicação para caracterizar os municípios: quem são e o que precisam para desenvolver ações de governo eletrônico e smart city. Revista de Administração Pública.

Renteria, C., Ramon Gil-Garcia, J., Pardo, T. A. (2019). Toward an Enabler- Based Digital Government Maturity Framework: A Preliminary Proposal Based on Theories of Change. In: Proceedings of the 12th International Conference on Theory and Practice of Electronic Governance (ICEGOV2019), 10 p.

SISP. (2017). Guia de Governança de TIC. Ministério do Planejamento, Desenvolvimento e Gestão. Disponível em: http://www.sisp.gov.br/.

UFMT (2019a).Plano Diretor De Tecnologia da Informação e Comunicação ～(PDTIC) 2020-2022.

Disponível

em: https://www1.ufmt.br/pdtic/arquivos/7fd0e4e898ad67ed81278656e2f1cc08.pdf.

. (2019b).Plano De Desenvolvimento Institucional (PDI) 2019-2023. Disponível em: http://www1.ufmt.br/pdiufmt/arquivos/a4e4a963aab7dc8d8cc561b45a1eb965.pdf

Yin, R. K. (2015). "Estudo de caso: planejamento e métodos", 5a ed. Porto Alegre, RS: Bookman. 